\title{
50 Years of Physical Review C: Probing the Secrets of Nuclei
}

\section{Researchers look back at key contributions to the field of nuclear physics.}

Physical Review C-the Physical Review journal dedicated to nuclear physics-was born fifty years ago.

To commemorate the anniversary, we asked the journal's editors to select a handful of papers that made key contributions to the field. We then tracked down some of the papers' authors, as well as earlier-career researchers now active in those areas, to get a glimpse into how each paper shaped the field and continues to do so today.

This article is the last in a four-part series commemorating the 50th anniversary of four Physical Review journals. Check out the other articles in the series to learn about some of the last five decades' biggest ideas in atomic and molecular physics, optics, and quantum information (Physical Review $A$ ); condensed matter and materials (Physical Review B); and particles, fields, gravitation, and cosmology (Physical Review $D$ ).

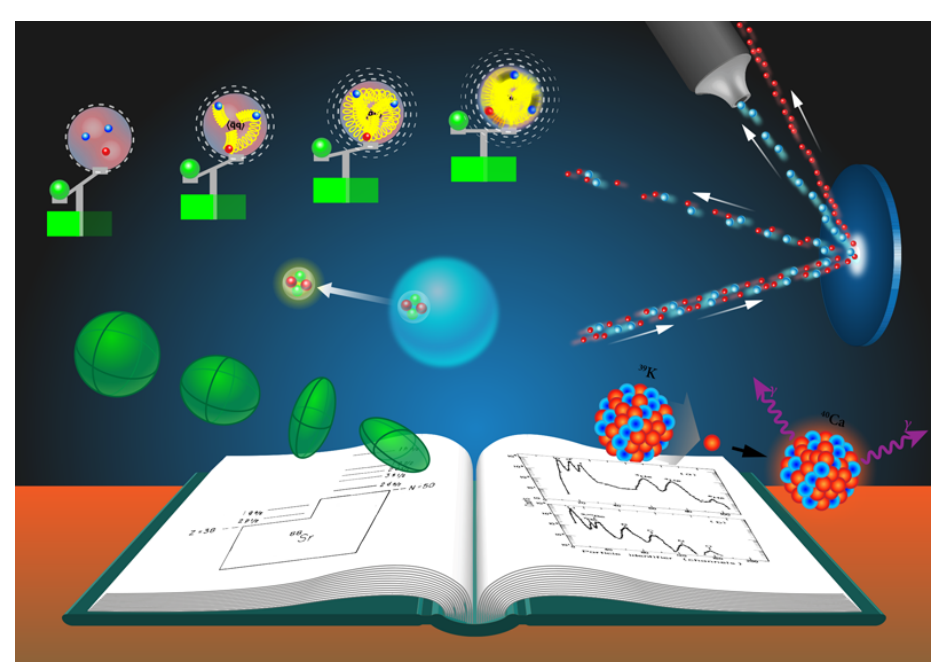

Credit: APS/Alan Stonebraker
-Erika K. Carlson

\section{9: A Universal Picture of Deformed Nuclei}

Atomic nuclei aren't always spherical; some naturally "deform" into an elongated shape, like an American football. In a 1979 paper, Stuart Pittel of the University of Delaware and Pedro Federman, then of the National Autonomous University of Mexico, showed how crucial a particular nuclear interaction is to driving nuclear deformation across the periodic table.

By the 1970s, physicists had determined that long-range interactions between the quadrupole fields of two nucleons were the likely cause of deformation in light nuclei-those with about 20 or so nucleons. They had also reached similar conclusions about some heavy nuclei in the rare-earth and actinide regions of the periodic table. In the lighter nuclei, there was evidence that interactions between unlike nucleons-a neutron and a proton-drove the deformation. But it wasn't clear whether this was the case for heavier nuclei. It also wasn't known whether nuclear deformation in intermediate-mass nuclei happened through the same physical mechanism.

Around the same time, experimentalists were discovering new examples of deformed nuclei in isotopes in the intermediate-mass range, with about 100 or so nucleons, such as those of zirconium and molybdenum. These discoveries in part inspired Federman and Pittel to tackle the nuclear deformation problem. "We had this idea that we could take what we knew from lighter nuclei and apply it in this new region of experimental observation," says Pittel.

The two worked out how quadrupole-quadrupole interactions would apply to nuclei in the intermediate-mass range and performed calculations to demonstrate how their ideas described the deformation behaviors of zirconium and molybdenum isotopes. Ultimately, the pair showed that these 
interactions between neutrons and protons are primarily responsible for causing nuclear deformation, and that the same physical explanation holds across the periodic table.

"This is textbook physics today in terms of understanding the basic source of deformation in systems across the nuclear chart, but this paper was really the first to spell it out so clearly," says Heather Crawford of Lawrence Berkeley Laboratory in California. An experimentalist, Crawford studies the nuclear structure of exotic isotopes using radioactive ion beams at various facilities, trying to understand further details of when and how deformation arises.

Crawford says that, in many aspects, today's researchers are pursuing questions very similar to those posed by Pittel and Federman in their paper. "We're just looking in different regions of the chart and seeing if the models that we have are really predicting structure, which is where we want to be, or if we still need more data to refine the details of those models."

\section{P. Federman and S. Pittel, "Unified shell-model description of} nuclear deformation,” Phys. Rev. C 20, 820 (1979).

\section{7: The Beginnings of Compressed Nuclear Matter Research}

When heavy nuclei collide at high speeds, do they pass through one another like bubbles of gas or splash off of one another in a spray of particles, like droplets of water? A team of researchers in the US and Germany addressed this question in the 1970s and 1980 s with a series of experiments, the first of which was reported in 1977.

Some theorists had predicted that a collision of heavy nuclei would produce nuclear matter with collective behavior-meaning the matter would splash off to the sides from the point of impact, like a liquid. In the 1977 paper's experiments, the researchers used a particle accelerator at Lawrence Berkeley Laboratory, called the Bevalac, to bombard gold and uranium targets with helium, carbon, and neon nuclei. They then measured the momentum distributions of particles that came off of the collision to see whether they seemed to follow the predicted splash-like behavior.

The initial experiments only measured particles at certain angles relative to the collision, so it was difficult to conclude

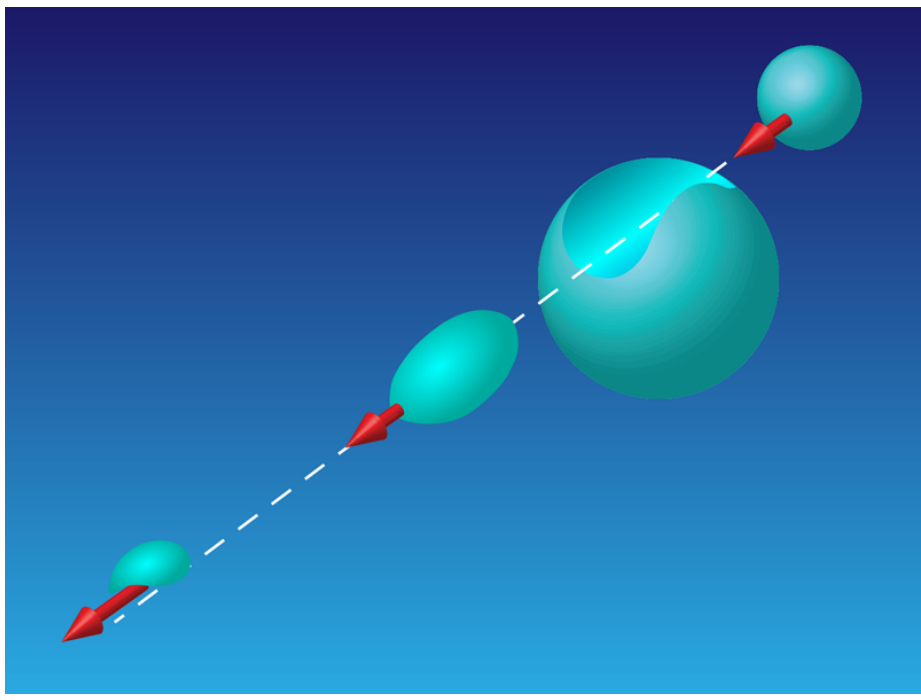

This image from the paper by Arthur Poskanzer and colleagues depicts one nucleus colliding into and scooping material out of another.

Credit: J. Gosset et al., Phys. Rev. C (1977)

whether the particles were following the predicted behavior. But a few years later, the team used a spherical detector called the Plastic Ball spectrometer, which was able to capture almost all particles produced in a given collision event. That way, researchers could get a fuller picture of the behavior of the nuclear matter, postcollision. And indeed, the later experiments clearly showed the splash-like collective behavior of the resultant particles.

These high-energy collision experiments melt parts of the nuclei into a dense pulp of protons and neutrons called compressed nuclear matter, similar to what exists in exotic astronomical objects like supernovae and neutron stars. "This was the beginning of making compressed nuclear matter," says Arthur Poskanzer, then of Lawrence Berkeley Laboratory and one of the 1977 paper's authors. Today, researchers are colliding even heavier nuclei at even higher energies. "At our energies, the protons and the neutrons get destroyed too, and we are looking really at a fireball of quarks and gluons," says Alice Ohlson of Lund University in Sweden.

Ohlson works on the ALICE experiment at CERN, which is dedicated to heavy ion collisions. The purpose of the 


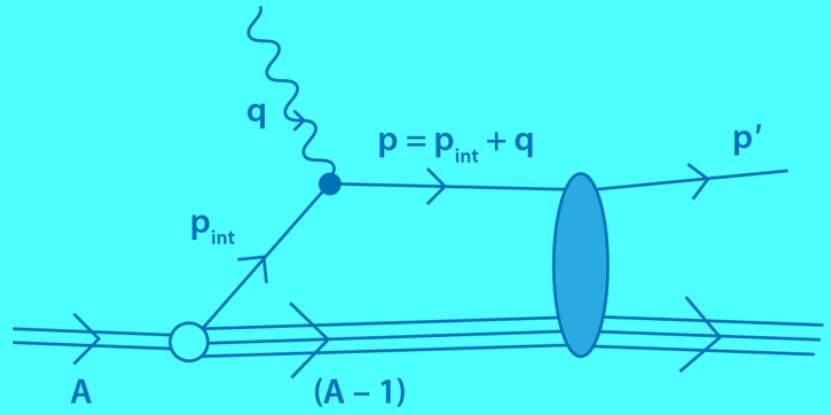

A scattering diagram from the paper by Mark Strikman and colleagues.

Credit: L. L. Frankfurt et al., Phys. Rev. C. (1993)

experiment is to study the quark-gluon plasmas that form from these extreme collisions and see what can be learned about the strong force, quantum chromodynamics (QCD), and the phase transitions of nuclear matter.

"There was a line in [the paper] about trying to use these measurements to understand the phase diagram of QCD, or the phase diagram of nuclear matter," Ohlson says. "And that's still what we're trying to do today."

J. Gosset et al., "Central collisions of relativistic heavy ions," Phys. Rev. C 16, 629 (1977).

\section{3: Detecting Overlapping Nucleons}

The nucleons in atomic nuclei experience many interactions, including so-called short-range correlations. This type of interaction occurs when two nucleons are so close together that they touch and acquire high momenta relative to each other-much larger than the average momentum of the nucleons in the nucleus. Many physicists thought that it would not be possible to directly observe short-range correlations experimentally because other nuclear phenomena would hide their effect. In a 1993 paper, Leonid Frankfurt of Tel Aviv University and colleagues showed the first experimental evidence of the elusive interactions.
Even decades before, physicists recognized that short-range correlations were likely a necessary component of nuclear interactions. But there wasn't yet an experiment capable of observing these correlations' effects; physicists needed a high-energy, high-momentum probe that didn't yet exist. After the 1970 s and 80 s, many researchers switched to using mean-field models that left out short-range correlations. "At this point, people were starting to say that it was simply impossible to measure short-range correlations in nuclei experimentally," says Mark Strikman of Penn State University, one of the paper's authors.

Eventually, a high-energy electron probe potentially capable of detecting short-range correlations in nuclei became available at SLAC National Accelerator Laboratory. However, the initial analysis of the scattering measurements ignored the dynamics of two-nucleon short-range correlations, considering only larger-scale, mean-field effects. But in the 90s, Frankfurt and colleagues figured out how to effectively analyze data from experiments with nuclei containing more than two nucleons and how to measure the number of nucleon pairs experiencing short-range correlations in a given nucleus.

"That paper was the first one that gave us an experimental handle on how many correlated pairs of nucleons we have inside the nucleus," says Or Hen of the Massachusetts Institute of Technology. By using high-energy scattering experiments, Hen studies short-range correlations and their implications for nucleon structure and neutron stars.

\section{L. Frankfurt et al., "Evidence for short-range correlations} from high $Q^{2}$ (e,e') reactions," Phys. Rev. C 48, 2451 (1993).

-Erika K. Carlson is a Corresponding Editor for Physics based in New York City. 